\title{
BORDERLINE PERSONALITY DISORDER DURING COVID-19 PANDEMIC
}

\author{
Dearisa Surya Yudhantara ${ }^{1}$; Ratri Istiqomah ${ }^{2}$ \\ Correspondence: drdsyudhantara.fk@ub.ac.id \\ ${ }^{I}$ Psychiatry Department Medical Faculty University of Brawijaya, Indonesia; ${ }^{2}$ SMF Psychiatry RSUD Dr. Saiful Anwar Malang, Indonesia
}

\begin{abstract}
Individuals with pre-existing mental problems are more likely to relapse or experience a recurrence of symptoms during The COVID-19 pandemic. This vulnerability can also manifest in those who suffer from Borderline Personality Disorders (BPD). Pandemic conditions significantly restrict people's social interaction for an extended period of time, resulting in severe restrictions on daily living routines and social isolation. When social interaction is restricted, emotional dysregulation and difficulty reading others' emotional expressions may lead borderline patients to anticipate subtle emotional expressions of fear or anxiety in their significant others, eliciting intense reactions such as outbursts of anger, increased irritability, and impulsive behaviors. Patients with personality disorders are more likely to experience crisis during a pandemic, which can result in self-inflicted injury or suicide. Psychotherapeutic interventions to support individuals with BPD who seek secondary mental health care include Dialectical Behavior Therapy (DBT), Mentalization-Based Therapy (MBT), and Schema-Focused Therapy (SFT), in addition to other briefer skills-based group therapy sessions, many of which have been made difficult to obtain since the COVID-19 pandemic began. Dialectical Behavioral Therapy (DBT) is one of the most effective psychotherapies for borderline personality disorder (BPD). With the adoption of social distancing and the elimination of face-to-face activities, there are challenges to DBT. DBT delivered via telemedicine and telehealth are pragmatic alternatives that have had varying degrees of success during this pandemic.
\end{abstract}

Keywords: borderline, personality, psychotherapy, COVID-19.

\section{Article History:}

Received: August 31, 2021

Accepted: September 25, 2021

Published: September 30, 2021
Cite this as: Yudhantara, D.S. Istiqomah, R. Borderline personality disorder during covid-19 pandemic Journal of Psychiatry Psychology and Behavioral Research; 2021.Vol. 2, Issue 2, p:14-17.

\section{INTRODUCTION}

COVID-19 is a global coronavirus disease pandemic that is still occurring. It is caused by severe acute respiratory syndrome coronavirus 2 (SARS-CoV-2). On March 11, 2020, the World Health Organization (WHO) announced a pandemic. This pandemic affects many aspects of life, including mental health conditions in individuals with mental disorders. ${ }^{1}$ The COVID-19 pandemic has resulted in anxiety, depression, and post-traumatic stress disorder symptoms in a range of population groups, including healthcare workers, the general public, patients, and quarantined individuals. ${ }^{2}$ Individuals who have been diagnosed with a mental illness in the past have a greater susceptibility to relapse or reappearance of symptoms during this pandemic. ${ }^{3}$

This vulnerability can also appear in people with Borderline Personality Disorders (BPD). Both in the general community and in clinical settings, BPD is frequent. BPD is estimated to have a point prevalence of 1.6 percent and a lifetime prevalence of 5.9 percent in large nationally representative nonclinical surveys of the general population in the United States. ${ }^{4,5}$ Cluster B personality disorders, such as BPD, are characterized by unstable interpersonal connections as well as overly emotional, impulsive, dramatic, and erratic behavior. It is possible that their vulnerability is caused by an underlying hyperresponsiveness to stress and hypersensitivity to stressors. ${ }^{6,7}$ Numerous difficulties experienced by people with BPD, such as affective dysregulation, chronic feelings of emptiness, fear of abandonment, and interpersonal dysfunction, are manifestations of an impaired capacity for self-actualization caused by early emotional neglect by significant attachment figures and subsequent life adversity. ${ }^{8}$

\section{METHOD}

This review take some journal who related with this topic.

\section{DISCUSS}

\section{Changes in Clinical Condition}

Pandemic situations cause people to substantially restrict their social interaction for an extended period of time, with severe limitations for everyday living routines and social isolation. Persons with BPD being compelled to maintain a distance from important others (friends, parents, and love ones) may be very detrimental. Individual with borderline personality disorders share an intense desire for emotional and physical closeness with others. During an outbreak, patients may experience diminished psychological well-being, overestimate the the 
pandemic's duration, and show little concern for the community. ${ }^{9}$ The rising sense of abandonment activated the underlying weaknesses of BPD, which were exacerbated by the current need for extensive indoor confinement and social isolation. The spinning funnel of emptiness and utter fear has the ability to magnify negative feelings and thoughts about oneself and others. ${ }^{10}$

It is also as explained that in patients with BPD, there are often problems in terms of mentalization. Patients can switch quickly from controlled to automatic mentalization from being overly sensitive to interpersonal stressors that arise during a pandemic situation. It may be related to the failure of others to reach him due to the limitations of the quarantine period. This can increase his internal stressors such as uncontrollable thoughts and can cause emotional crises. ${ }^{10}$ Patients with BPD frequently run out of psychological energy when confronted with extensive social relationships; however, the prospect of limiting social relationships can be stressful as well. Negative self- and other-images can logically develop as a result of social isolation. It is likely that social separation will be regarded incorrectly as neglect, disinterest, or a sense of abandonment. ${ }^{9}$ When social interaction is restricted, emotional dysregulation including emotional crises as well as difficulty in reading other people's emotional expressions (mentalization) may cause borderline patients to anticipate subtle emotional signs of fear or anxiety in their significant others, which can lead to extreme emotions like rage, irritation, and impulsive behavior. ${ }^{11}$

According to a study conducted in Spain, there was no significant clinical difference in patients with BPD 2.5 months after the pandemic began. However, it is also stated that in general social isolation has a negative effect on patients. However, this negative effect of isolation will be more pronounced in patients with BPD because of their preexisting clinical susceptibility. ${ }^{12}$ In one of France's studies on this subject, daily analysis using the Beck Hopelessness Scale and the Beck Depression Inventory revealed that confinement strongly predicted a decrease in feelings of shame or guilt, dread, and anxiety. During the same period, on the other hand, there was a rise in distress. Weekly monitoring of problem behaviors revealed a drop in binge-eating habits, as well as a trend toward a decrease in alcohol intake while in confinement, according to the findings. It is possible that the slower pace of daily social and professional life is responsible for these improvements. ${ }^{13}$

A survey-based study conducted in Norway in the early wave of the pandemic showed a significant increase in symptoms of anxiety and depression compared to before the pandemic. About a quarter to a third of respondents also reported increased verbal and physical aggression. There has also been an increase in substance and alcohol use. Regarding childcare and work, patients with BPD also reported negative effects of the pandemic. ${ }^{14}$

\section{Self-Harm and Suicidal Behavior-Suicide Attempt}

Both the subjective experience of being alone (i.e., loneliness) and the objective circumstance (e.g., living alone) were substantially connected with suicidal consequences in BPD and had a significant impact on suicidal thoughts and attempt. ${ }^{15}$ Existing medical problems also play a role in the emergence of suicidal thoughts. During a pandemic, it is not only a matter of limited mental health care, but also other general medical health problems. For example, surgery or other medical procedures that have been delayed. This means that they have to wait and live through prolonged pain that can drive them to commit suicide. This will be even more detrimental when there is difficulty in accessing mental health services. ${ }^{16}$ In one study after adjusting for demographic variables and clinical conditions, there were two diagnostic criteria for BPD that were specific to suicidal thoughts and behavior. The two symptoms were self-injurious behavior and chronic emptiness which were found to be significantly linked to a higher risk of suicide. Therefore, these two symptoms become important clinical markers in BPD patients that lead to an assessment of suicide risk. ${ }^{17}$

During a pandemic, self-harm and suicidal behavior in people with personality problems varies depending on the study. A study conducted in the United Kingdom examined emergency department visits and discovered an increase in the incidence of self-injury or self-harm when compared to pre-pandemic levels. The increase in self-harm is primarily due to drug overdose ${ }^{18}$ because patients with borderline personality who perceive distance from others as abandonment may be more inclined to self-medicate with substances and engage in suicide and non-suicidal self-harm activities to cope with loneliness. ${ }^{9}$ Another study found no increase in self-harm or suicidal behavior during the pandemic in comparison to the prepandemic period. The study does warn, however, that patients with personality disorders are more prone to experience crisis during a pandemic. Additionally, the study showed that people with personality disorders who were predisposed to suicidal behavior earlier stated that similar behaviors reappeared following the epidemic. ${ }^{14}$ However, one guiding idea is that suicide is preventable and that immediate action must be done to safeguard people's mental health, especially in patients with BPD. ${ }^{16}$

\section{Access to Psychotherapy Services}

Psychotherapeutic interventions for individuals with BPD seeking secondary mental health care include Dialectical Behavior Therapy (DBT) (Linehan et al., 1993), SchemaFocused Therapy (SFT), ${ }^{20}$ and Mentalization-Based Therapy (MBT) [21], in addition to other shorter skills-based group treatment sessions, the availability of which has been increasingly challenging since the COVID-19 pandemic began. ${ }^{21,22}$

Thirty years have elapsed since the creation of MentalizationBased Treatment (MBT), which was originally intended to treat patients with BPD, but is now being used to treat a variety of other psychiatric disorders. According to the mentalization approach to BPD, impairments in social cognition, namely a weakened ability to understand oneself and others in terms of mental processes, play a significant role in the development of the condition and its manifestation. Long distance approach or over the phone are often obstacles to the implementation of this type of therapy. ${ }^{21}$

Dialectical Behavioral Therapy (DBT) is one of the definitive psychotherapies prescribed for BPD. DBT is primarily used to address symptoms associated with emotional dysregulation in mental disorders. Prior to the epidemic, this treatment was delivered in person, emphasizing mindfulness exercises, emotional management, distress tolerance, and interpersonal skills. Coaching by telephone is possible in some circumstances. $^{23}$

There are mixed results and evidence of the effectiveness of tele-mental health in relation to psychiatric symptoms. Online discussion groups/forums, video conferencing, smartphone applications, text messages, and email can all be helpful modes 
of communication for the delivery of mental health treatments. However, in conditions that have limitations on physical meetings such as during this pandemic, it must be optimally pursued by other possible ways. This is because people with mental health problems or mental disorders will continue to or even increasingly need mental health services that are easily accessible. $^{24}$

Modification of therapy in COVID-19 pandemic era by telephone is more often carried out than therapy with digital media. The age of the patient who is familiar with using this therapy modification (telephone or digital media) is mostly at a young age. Although some patients doubted the benefits of telemedicine or remote therapy, some felt that they could still benefit from this kind of therapy. Although under certain conditions, they feel that something is missing because they cannot meet the therapist in person or face-to-face. ${ }^{14}$

There are barriers to DBT with the implementation of social distancing and the reduction of face-to-face activities. The majority of DBT activities are conducted online by telephone, videoconference, or email. At times, face-to-face meetings with patients may be limited. This condition is believed to be incapable of delivering the full therapeutic impact. Patients and therapists report varied degrees of success. Patients and therapists alike are uneasy about engaging in psychotherapy online, and some believe that this is a temporary measure until the prohibition on face-to-face meetings is repealed. ${ }^{25}$

\section{CONCLUSION}

The COVID-19 pandemic has had a significant impact on patients diagnosed with Borderline Personality Disorder (BPD). Although the form of symptoms may vary according to the patient's condition, some clinical conditions that were previously calm or within the baseline range may worsen or emerge as a result of a pandemic situation. Situations that have the potential to cause stress involve stressors that occur both interpersonally and within the individual himself. Symptoms that require special attention in BPD patients are self-harm, suicidal behavior or even attempted suicide. As we know that psychotherapy approach is the main therapy for BPD patients. In this pandemic period that limits physical encounters, solutions must still be found so that BPD patients can still get the therapy they need. In this case, DBT psychotherapy is said to be possible through telemedicine. This is a good opportunity for the patient to be able to maintain his mental state in a better condition.

\section{REFERENCES}

1. CDC, "Coping with Stress," 2020, 2020.

2. Y. Luo, C. R. Chua, Z. Xiong, R. C. Ho, and C. S. H. Ho, "A Systematic Review of the Impact of Viral Respiratory Epidemics on Mental Health: An Implication on the Coronavirus Disease 2019 Pandemic," Front. Psychiatry, vol. $0, \quad$ p. 1247, Nov. 2020, doi: 10.3389/FPSYT.2020.565098.

3. C. Moreno et al., "How mental health care should change as a consequence of the COVID-19 pandemic," The Lancet. Psychiatry, vol. 7, no. 9, p. 813, Sep. 2020, doi: 10.1016/S2215-0366(20)30307-2.

4. M. Lenzenweger, M. Lane, A. Loranger, and R. Kessler, "DSM-IV personality disorders in the National Comorbidity Survey Replication," Biol. Psychiatry, vol.
BPD patients who are unable to see a therapist due to limitations during the pandemic may experience feelings of uncertainty, misperceptions, misunderstandings, and even paranoid ideas that are usually associated with stress. In patients who have previously undergone psychotherapy, there may be negative effects during this pandemic. Realizing that they are not currently visible to the therapist due to limited physical encounters can make it difficult for patients to fully engage in the psychotherapy that is actually on going. For example, there is a change in behavior or skill that was previously assigned and is usually reported and observed by the therapist. Problems like this can be handled and solved specifically by using distance psychotherapy formats such as telemedicine, although not as optimal as face-to-face physical meetings. $^{14}$

Telemedicine and telehealth are sensible solutions during this pandemic. The most commonly encountered form of obstacle during the shift to telehealth is technological one on both the provider/therapist and patient side. The majority of providers/therapists, on the other hand, stated that their experience exceeded their expectations and that they had a favorable assessment of patient acceptance. The most challenging modality to give via telehealth is group therapy. Numerous benefits of DBT administered via telehealth/telemedicine have been highlighted, including the removing limitations to care, such as distance, transportation challenges, and caregiver and work responsibilities.$^{26}$

62, no. 6, pp. 553-564, Sep. 2007, doi: 10.1016/J.BIOPSYCH.2006.09.019.

5. B. Grant et al., "Prevalence, correlates, disability, and comorbidity of DSM-IV borderline personality disorder: results from the Wave 2 National Epidemiologic Survey on Alcohol and Related Conditions," J. Clin. Psychiatry, vol. 69, no. 4, pp. 533-545, 2008, doi: 10.4088/JCP.V69N0404.

6. K. Wingenfeld, C. Spitzer, N. Rullkötter, and B. Löwe, "Borderline personality disorder: Hypothalamus pituitary adrenal axis and findings from neuroimaging studies," Psychoneuroendocrinology, vol. 35, no. 1, pp. 154-170, Jan. 2010, doi: 10.1016/J.PSYNEUEN.2009.09.014.

7. K. Bertsch et al., "Neural correlates of emotional action control in angerprone women with borderline personality disorder," J. Psychiatry Neurosci., vol. 43, no. 3, pp. 161170, May 2018, doi: 10.1503/JPN.170102.

8. A. Bateman and P. Fonagy, "Mentalization-Based Treatment for Personality Disorders," Ment. Treat. Personal. Disord., Aug. 2016, doi: 10.1093/MED:PSYCH/9780199680375.001.0001.

9. E. Preti, R. Di Pierro, E. Fanti, F. Madeddu, and R. Calati, "Personality Disorders in Time of Pandemic," Curr. Psychiatry Rep., vol. 22, no. 12, Dec. 2020.

10.S. C. Chong, "Psychological impact of coronavirus outbreak on borderline personality disorder from the perspective of mentalizing model: A case report," Asian J. Psychiatr., vol. 52, Aug. 2020.

11. T. R. Lynch, M. Z. Rosenthal, D. S. Kosson, J. S. Cheavens, C. W. Lejuez, and R. J. R. Blair, "Heightened sensitivity to facial expressions of emotion in borderline personality disorder," Emotion, vol. 6, no. 4, pp. 647-655, Nov. 2006, doi: 10.1037/1528-3542.6.4.647.

12.F. Álvaro et al., "Clinical course and predictors in patients with borderline personality disorder during the COVID-19 outbreak: A 2.5-month naturalistic exploratory study in Spain," Psychiatry Res., vol. 292, Oct. 2020, doi: 10.1016/J.PSYCHRES.2020.113306. 
13. V. Salamin et al., "Adaptations of an outpatient Dialectical Behavioral Therapy during the COVID-19 pandemic and consequences of the confinement on patients with borderline personality disorder," Ann. Med. Psychol. (Paris)., vol. 179, no. 2, pp. 131-136, Feb. 2021, doi: 10.1016/J.AMP.2020.08.006.

14. E. Hartveit Kvarstein et al., "Vulnerability of personality disorder during COVID-19 crises: a multicenter survey of mental and social distress among patients referred to treatment.," Nord. J. Psychiatry, pp. 1-12, Aug. 2021.

15. R. Calati et al., "Suicidal thoughts and behaviors and social isolation: A narrative review of the literature," J. Affect. Disord., vol. 245, pp. 653-667, Feb. 2019, doi: 10.1016/J.JAD.2018.11.022.

16. A. John, J. Pirkis, D. Gunnell, L. Appleby, and J. Morrissey, "Trends in suicide during the covid-19 pandemic," $B M J$, vol. 371, Nov. 2020, doi: 10.1136/BMJ.M4352.

17.C. M. Grilo and T. Udo, "Association of Borderline Personality Disorder Criteria With Suicide Attempts Among US Adults + Supplemental content," JAMA Netw. Open, vol. 4, no. 5, p. 219389, 2021, doi: 10.1001/jamanetworkopen.2021.9389.

18. N. Henry, S. Parthiban, and A. Farroha, "The effect of COVID-19 lockdown on the incidence of deliberate selfharm injuries presenting to the emergency room," Int. $J$. Psychiatry Med., vol. 56, no. 4, pp. 266-277, Jul. 2021, doi: 10.1177/0091217420982100.

19. M. Linehan, H. Heard, and H. Armstrong, "Naturalistic follow-up of a behavioral treatment for chronically parasuicidal borderline patients," Arch. Gen. Psychiatry, vol. 50, no. 12, pp. 971-974, 1993, doi: 10.1001/ARCHPSYC.1993.01820240055007.

20.J. Young, Cognitive therapy for personality disorders: A schema-focused approach, 3rd ed. - PsycNET. Professional Resource Press/Professional Resource Exchange, 1999.

21. A. Bateman and P. Fonagy, "Psychotherapy for Borderline Personality Disorder: Mentalization Based Treatment." 2004.

22. J. McLoughlin, M. M. O'Grady, and B. Hallahan, “Impact of the COVID-19 pandemic on patients with pre-existing mood disorders," Ir. J. Psychol. Med., pp. 1-10, Apr. 2021, doi: 10.1017/IPM.2021.38.

23. M. M. Linehan, "DBT Skills Training Manual, Second Edition," DBT Ski. Train. Manual, Second Ed., pp. 3-19, 2015.

24.X. Zhou et al., "Opinion The Role of Telehealth in Reducing the Mental Health Burden from COVID-19," doi: 10.1089/tmj.2020.0068.

25. R. Lakeman and J. Crighton, "The Impact of Social Distancing on People with Borderline Personality Disorder: The Views of Dialectical Behavioural Therapists," Issues Ment. Health Nurs., vol. 42, no. 5, pp. 410-416, 2021, doi: 10.1080/01612840.2020.1817208.

26. S. J. Landes, J. A. Pitcock, M. S. Harned, S. L. Connolly, L. L. Meyers, and C. M. Oliver, "Provider perspectives on delivering dialectical behavior therapy via telehealth during COVID-19 in the Department of Veterans Affairs.," Psychol. Serv., Aug. 2021. 